M. Á. Barja and F. Zucconi

Nagoya Math. J.

Vol. 164 (2001), 103-131

\title{
ON THE SLOPE OF FIBRED SURFACES
}

\author{
MIGUEL ÁNGEL BARJA ${ }^{1}$ AND FRANCESCO ZUCCONI ${ }^{2}$
}

\begin{abstract}
We give an asymptotically sharp lower bound for the slope $\lambda(f)$ of a fibration $f: S \rightarrow B$, where $S$ is a surface and $B$ is a curve, if there exists an involution on the general fibre $F$ of $f$. We also construct a new lower bound of $\lambda(f)$ depending increasingly on the irregularity of $S$; as an application of this new bound we have a criteria to control the existence of other fibrations on $S$.
\end{abstract}

\section{$\S 0$. Introduction}

Let $f: S \rightarrow B$ be a surjective holomorphic map with connected fibre $F$ from a complex smooth projective surface $S$ onto a complex smooth curve $B$. We always assume that it is relatively minimal, i.e., that there is no $(-1)$-rational curve contained in a fibre of $f$. Following [6, p.9] we call $f$ a minimal fibration or a minimal genus $b$ pencil of curves of genus $g$ where $g=g(F)$ and $b=g(B)$. It is called smooth if all its fibres are smooth, isotrivial if all its smooth fibres are reciprocally isomorphic, and locally trivial if it is smooth and isotrivial.

Our results enable to study the geographical problem of $f$ (that is, to relate numerical invariants of $F, S$ and $B$ ) through the control of some geometrical properties of the general fibre $F$ or by the influence of some global properties of $S$ such as the relative irregularity $q_{f}=q-b$. Now we recall the basic relative invariants for $f$. If $V$ is an n-dimensional smooth variety, $\omega_{V}=\mathcal{O}_{V}\left(K_{V}\right)$ is the invertible sheaf generated by $d x_{1} \wedge \ldots \wedge d x_{n}$, where $x_{1}, \ldots, x_{n}$ are local coordinates and $K_{V}$ is called the canonical divisor. Let $p_{g}=h^{0}\left(S, \omega_{S}\right), q=h^{1}\left(S, \omega_{S}\right), \chi \mathcal{O}_{S}=p_{g}-q+1$ and let $e(X)$ be the topological Euler characteristic of $X$. Then we consider the following set of

Received March 7, 2000.

1991 Mathematics Subject Classification: Primary 14H10, Secondary 14J10.

${ }^{1}$ Miguel Ángel Barja has been partially supported by CICYT PS93-0790 and HCM project n.ERBCHRXCT-940557.

${ }^{2}$ Francesco Zucconi has been partially supported by HCM project n.ERBCH RXCT-940557. 
relative invariants:

$$
\begin{aligned}
& K_{S / B}^{2}=\left(K_{S}-f^{*} K_{B}\right)^{2}=K_{S}^{2}-8(b-1)(g-1) \\
& \chi_{f}=\operatorname{deg} f_{*} \omega_{S / B}=\chi \mathcal{O}_{S}-(b-1)(g-1) \\
& e_{f}=e(S)-e(B) e(F)=e(S)-4(b-1)(g-1) .
\end{aligned}
$$

We have the following classical results:

TheOrem 0.1. Let $f: S \rightarrow B$ be a minimal genus $b$ pencil of curves of genus $g \geq 2$. Then

(i) (Noether) $12 \chi_{f}=e_{f}+K_{S / B}^{2}$.

(ii) (Zeuthen-Segre) $e_{f} \geq 0$. Moreover, $e_{f}=0$ if and only if $f$ is smooth.

(iii) (Arakelov) $K_{S / B}^{2} \geq 0$. Moreover, $K_{S / B}^{2}=0$ only if $f$ is isotrivial.

(iv) $\chi_{f} \geq 0$. Moreover, $\chi_{f}=0$ if and only if $f$ is locally trivial.

When $f$ is not locally trivial, Xiao (cf. [17]) defines the slope of $f$ as

$$
\lambda(f)=\frac{K_{S / B}^{2}}{\chi_{f}} .
$$

It follows immediately from Noether's equality that $0 \leq \lambda(f) \leq 12$. We are mostly concerned with a lower bound of the slope. The main known result in this direction is:

Theorem 0.2. (Cornalba-Harris, Xiao) If $g \geq 2$ and $f$ is not locally trivial, then $\lambda(f) \geq 4-\frac{4}{g}$.

After that, the main task is to investigate the influence of some properties of the fibration on the behaviour of the slope. The first problem is to study the influence of the relative irregularity $q_{f}=q(S)-b$. The main known result is:

Theorem 0.3. (Xiao) If $q>b$ then $\lambda(f) \geq 4$. If $\lambda(f)=4$ and $q>b$ then $q=b+1$ and $f_{*} \omega_{S / B}$ is semistable. 
The other most considered problem is the study of how properties of the general fibre $F$ influence. Mostly due to the work of Konno (cf. [11],[13]; see also [7] and [16] for other references) we know the Clifford index (or the gonality) of the general fibre has some meaning in the lower bound of the slope. There are evidences for this. For example, it is known that equality $\lambda(f)=4-\frac{4}{g}$ only holds when $F$ is hyperelliptic. When $F$ is trigonal, a better bound is known (see Remark 3.5). On the other hand the following theorem shows that if $g>>0$ and $F$ has general Clifford Index then $\lambda(f) \simeq 6$ and so, together with 0.3 , it forces to understand the case $q_{f}>0, g>>0$ and $4<\lambda(f)<6$.

TheOrem 0.4. (Harris-Eisenbud, Konno) Assume that $g$ is odd and that the general fibre $F$ is of general Cifford index. If $f$ is not a semistable fibration assume that Green's conjecture on syzygies of the canonical curves holds. Then

$$
\lambda(f) \geq 6 \frac{g-1}{g+1} .
$$

An explicit sharp lower bound for $\lambda(f)$ depending on the Clifford index of $F$ should not be easy and it should depend on other parameters. Indeed, when the fibre is trigonal, the behaviour of $\lambda(f)$ depends on the fact that $F$ is general or not in the set of trigonal curves (see [16]). A similar behaviour should hold for tetragonal fibrations: there are tetragonal fibrations with $\lambda(f)=4$ for any genus $g$ (see $[1]$ ), although the general fibre is always bielliptic.

In $\S 1$ we give an idea of Xiao's method, which is our main tool. In $\S 2$ we study the behaviour of $\lambda(f)$ when the general fibre $F$ is a double cover in such a way that extends to a double cover of the fibration itself: see definition 2.1. In $\S 3$ we explicit the influence of $q_{f}$ on $\lambda(f)$ through an increasing function on $q_{f}$ generalizing Theorem 0.3 . As natural by-product of this estimates and the previous theorems it seems possible to construct through $\lambda(f)$ a geography for fibrations as in the case of surfaces of general type.

We set aside the double cover case by two reasons: 1) all double covers are special curves of non general Clifford index; as it happens in the case of bielliptic curves with respect to tetragonal ones, fibrations which are double covers are candidates to be exceptional in the study of $\lambda(f) .2$ ) Xiao's method works very well if the possibility for $f$ to be a double cover 
is excluded, which also suggest the study of double covers as exceptional. For these fibrations we get:

TheOREM 0.5. Let $f: S \rightarrow B$ be a non isotrivial minimal genus $b$ pencil of curves of genus $g$ which is a double cover of a fibration of genus $\gamma$. If $g \geq 4 \gamma+1$ then

$$
\lambda(f) \geq 4+4 \frac{(\gamma-1)(g-4 \gamma-1)}{(g-4 \gamma-1)(g-\gamma)+2(g-1) \gamma^{2}} .
$$

In our paper there is also a refined version of this theorem but it is more complicate to state: see 2.4. Next theorem is a partial answer to a longstanding natural question: how special are fibrations with $\lambda(f)<4$ ?

ThEOREM 0.6. Let $f: S \rightarrow B$ be a non isotrivial minimal genus $b$ pencil of curves $F$ of genus $g$ which is a double cover of a fibration $\sigma: V \rightarrow$ $B$ with fibre $E$ of genus $\gamma$. If $F$ is not hyperelliptic nor tetragonal, $\gamma \geq 1$ and $g \geq 2 \gamma+11$ then $\lambda(f) \geq 4$.

Our next theorem gives a bound which is better than the bound in Theorem 0.3 if $g>>0$ and it gives an affirmative answer to the expected influence of $q_{f}$ on the slope. Moreover it gives a bound which is (strictly) increasing and it is asymptotically sharp (see Example 2.8).

TheOrem 0.7. Let $f: S \rightarrow B$ be a minimal genus b pencil of curves of genus $g$ which is not locally trivial and not a double cover fibration. Assume that $g=g(F) \geq 5$ and $q_{f}=q(S)-b \geq 1$ then the following bounds hold:

(i) If $q_{f} \geq 2$ and $g \geq \frac{3}{2} q_{f}+2$ then

$$
\begin{aligned}
& \lambda(f) \geq \frac{8 g(g-1)\left(4 g-3 q_{f}-10\right)}{8 g(g-1)\left(g-q_{f}-2\right)+3\left(q_{f}-2\right)(2 g-1)} \quad \text { if } F \text { is not trigonal } \\
& \lambda(f) \geq \frac{4 g(g-1)\left(4 g-3 q_{f}-10\right)}{4 g(g-1)\left(g-q_{f}-2\right)+(g-4)(2 g-1)} \quad \text { if } F \text { is trigonal. }
\end{aligned}
$$

(ii) If $g<\frac{3}{2} q_{f}+2$ then

$$
\lambda(f) \geq \frac{4 g(g-1)(2 g-7)}{\frac{4}{3} g(g-1)(g-3)+(g-4)(2 g-1)} .
$$


As an application we obtain a nice relation between $\lambda(f)$ and the existence of other fibration on $S$ onto curves of genus at least 2 (see also Corollaries 3.9, 3.10):

ThEOREM 0.8. Let $f: S \rightarrow B$ be a minimal genus b pencil of curves of genus $g$ which is not locally trivial and not a double cover fibration. Let $\mathcal{C}=\left\{\pi_{i}: S \rightarrow C_{i} \text { fibrations, } c_{i}=g\left(C_{i}\right) \geq 2, \pi_{i} \neq f\right\}_{i \in I}$, Assume $\mathcal{C} \neq \emptyset$ and let $c=\max \left\{c_{i} \mid i \in I\right\}$. If $q_{f}=q-b \geq 1$ (i.e., $f$ is not an Albanese fibration) then

$$
\lambda(f) \geq 4+\frac{c-1}{g-c} .
$$

Moreover if $\operatorname{dim} \operatorname{alb}(S)=1$ (then necessarily $b=0$ ) we have

$$
\lambda(f) \geq 4+\frac{q-1}{g-q} .
$$

\section{$\S 1 . \quad$ Preliminaries}

Here we give a brief run-down of Xiao's method to estimate $\lambda(f)$. Its method uses a result of Harder and Narasimhan and the theorem of Clifford.

Let $\mathcal{E}$ be a locally free sheaf on $B$ and let $\mathcal{G} \mathcal{R}(\mathcal{E})$ be the set of the locally free subsheaves of $\mathcal{E}$; it is defined a function $\mu: \mathcal{G} \mathcal{R}(\mathcal{E}) \rightarrow \mathbb{Q}, \mathcal{F} \mapsto$ $\mu(\mathcal{F})=\operatorname{deg}(\mathcal{F}) / \operatorname{rank}(\mathcal{F})$. We recall that $\mathcal{E}$ is Mumford-stable (respectively Mumford-semistable) if for every proper subbundle $\mathcal{F}$ of $\mathcal{E}, 0<\operatorname{rank}(\mathcal{F})<$ $\operatorname{rank}(\mathcal{E})$ we have

$$
\mu(\mathcal{F})<\mu(\mathcal{E})(\operatorname{resp} . \mu(\mathcal{F}) \leq \mu(\mathcal{E}))
$$

The Harder-Narasimhan theorem concerns the maximum value for $\mu$.

TheOREM 1.1. Let $\mathcal{E}$ be a locally free sheaf on a smooth curve $B$, there exists a unique filtration by subbundles

$$
0=\mathcal{E}_{0} \subset \mathcal{E}_{1} \subset \cdots \subset \mathcal{E}_{l}=\mathcal{E}
$$

such that, for $i=1, \ldots, l, \mathcal{E}_{i} / \mathcal{E}_{i-1}$ is the maximal semistable subbundle of $\mathcal{E} / \mathcal{E}_{i-1}$. We put $\mu_{i}=\mu\left(\mathcal{E}_{i} / \mathcal{E}_{i-1}\right)$. In particular for every $i=1, \ldots, l, \mathcal{E}_{i} / \mathcal{E}_{i-1}$ is the unique subbundle of $\mathcal{E} / \mathcal{E}_{i-1}$ such that for every subbundle $\mathcal{F}$ of $\mathcal{E} / \mathcal{E}_{i-1}$ we have $\mu(\mathcal{F}) \leq \mu_{i}$ and if $\mu(\mathcal{F})=\mu_{i}$ then $\mathcal{F} \subset \mathcal{E}_{i} / \mathcal{E}_{i-1}$. Moreover $\mu_{1}>$ $\mu_{2}>\ldots>\mu_{l}$.

Proof. See [9]. 
The numbers $\left\{\mu_{i}\right\}_{1 \leq i \leq l}$ are called the Harder-Narasimhan slopes of $\mathcal{E}$.

For lack of reference we shall prove the following result that relates the Harder-Narasimhan filtration of a direct sum to the filtrations of the summands.

Proposition 1.2. Let $\mathcal{E}, \mathcal{H}, \mathcal{K}$ be locally free sheaves on a smooth curve B. Let $0=\mathcal{E}_{0} \subset \mathcal{E}_{1} \subset \ldots \subset \mathcal{E}_{\ell}, 0=\mathcal{H}_{0} \subset \mathcal{H}_{1} \subset \ldots \subset \mathcal{H}_{\ell_{1}}, 0=$ $\mathcal{K}_{0} \subset \mathcal{K}_{1} \subset \ldots \subset \mathcal{K}_{\ell_{2}}$ be their Harder-Narasimhan filtrations and $\left\{\mu_{i}\right\}_{1 \leq i \leq \ell}$, $\left\{\mu_{i}^{\mathcal{H}}\right\}_{1 \leq i \leq \ell_{1}},\left\{\mu_{i}^{\mathcal{K}}\right\}_{1 \leq i \leq \ell_{2}}$ their Harder-Narasimhan slopes. Assume $\mathcal{H} \oplus \mathcal{K}=$ $\mathcal{E}$. Then we can define $\psi:\{0, \cdots, \ell\} \rightarrow\left\{0,1, \cdots, \ell_{1}\right\}, \phi:\{0, \cdots, \ell\} \rightarrow$ $\left\{0,1, \cdots, \ell_{2}\right\}$ such that

(i) $\psi(0)=\phi(0)=0$; for $1 \leq i \leq \ell, \psi(i)=\psi(i-1)$ if $\mu_{t}^{\mathcal{H}} \neq \mu_{i}$ for every $t \in\left\{1, \cdots, \ell_{1}\right\}$, (respectively, $\phi(i)=\phi(i-1)$ if $\mu_{s}^{\mathcal{K}} \neq \mu_{i}$ for every $\left.s \in\left\{1, \cdots, \ell_{2}\right\}\right)$ and $\psi(i)=t$ if $\mu_{t}^{\mathcal{H}}=\mu_{i}$, (respectively, $\phi(i)=s$ if $\left.\mu_{s}^{\mathcal{K}}=\mu_{i}\right)$;

(ii) $\mathcal{E}_{i}=\mathcal{H}_{\psi(i)} \oplus \mathcal{K}_{\phi(i)}$.

Proof. Call $\pi_{\mathcal{H}}: \mathcal{E} \rightarrow \mathcal{H}, \pi_{\mathcal{K}}: \mathcal{E} \rightarrow \mathcal{K}$ the natural projections. The sheaves $\mathcal{E}_{1}^{\mathcal{H}}=\pi_{\mathcal{H}}\left(\mathcal{E}_{1}\right), \mathcal{E}_{1}^{\mathcal{K}}=\pi_{\mathcal{K}}\left(\mathcal{E}_{1}\right)$ are locally free since they are torsion free $\left(\mathcal{E}_{1}^{\mathcal{H}} \subseteq \mathcal{H}, \mathcal{E}_{1}^{\mathcal{K}} \subseteq \mathcal{K}\right)$. We have $\mathcal{E}_{1} \subseteq \mathcal{E}_{1}^{\mathcal{H}} \oplus \mathcal{E}_{1}^{\mathcal{K}}$.

Assume $\mathcal{E}_{1}^{\mathcal{H}} \neq 0$. Since $\mathcal{E}_{1}$ is semistable and $\mathcal{E}_{1}^{\mathcal{H}}$ is a quotient, we have that $\mu\left(\mathcal{E}_{1}^{\mathcal{H}}\right) \geq \mu\left(\mathcal{E}_{1}\right)=\mu_{1}$. From the inclusions $\mathcal{E}_{1}^{\mathcal{H}} \subseteq \mathcal{H} \subseteq \mathcal{E}$ we get $\mu_{1} \leq$ $\mu\left(\mathcal{E}_{1}^{\mathcal{H}}\right) \leq \mu_{1}^{\mathcal{H}} \leq \mu_{1}$ since $\mathcal{H}_{1}, \mathcal{E}_{1}$ are the maximal unstabilizing sheaves in $\mathcal{H}$ and $\mathcal{E}$ respectively. Hence $\mu_{1}=\mu_{1}^{\mathcal{H}}$ and $\mathcal{E}_{1}^{\mathcal{H}} \subseteq \mathcal{H}_{1} \subseteq \mathcal{E}_{1}$ by the maximality of $\mathcal{H}_{1}$ and of $\mathcal{E}_{1}$ (see 1.1). The same argument works if $\mathcal{E}_{1}^{\mathcal{K}} \neq 0$.

Assume $\mu_{1}^{\mathcal{K}} \neq \mu_{1}$. Then necessarily $\mathcal{E}_{1}^{\mathcal{K}}=0$ and $\mathcal{E}_{1}^{\mathcal{H}} \neq 0$. Hence $\mu_{1}^{\mathcal{H}}=\mu_{1}$, $\mathcal{E}_{1} \subseteq \mathcal{E}_{1}^{\mathcal{H}} \subseteq \mathcal{F}_{1}$ and then $\mathcal{E}_{1}=\mathcal{F}_{1}=\mathcal{H}_{1} \oplus \mathcal{K}_{0}$ by maximality. The same argument works if $\mu_{1}^{\mathcal{H}} \neq \mu_{1}$.

Assume $\mu_{1}^{\mathcal{H}}=\mu_{1}^{\mathcal{K}}=\mu_{1}$. Then $\mathcal{E}_{1} \subseteq \mathcal{E}_{1}^{\mathcal{H}} \oplus \mathcal{E}_{1}^{\mathcal{K}} \subseteq \mathcal{H}_{1} \oplus \mathcal{K}_{1}$ with $\mu\left(\mathcal{H}_{1} \oplus\right.$ $\left.\mathcal{K}_{1}\right)=\mu_{1}$. Again by maximality of $\mathcal{E}_{1}$ we conclude $\mathcal{E}_{1}=\mathcal{H}_{1} \oplus \mathcal{K}_{1}$.

Now the proof follows by induction dealing with $\mathcal{E} / \mathcal{E}_{1}=\mathcal{H} / \mathcal{H}_{\psi(1)} \oplus$ $\mathcal{K} / \mathcal{K}_{\phi(1)}$.

COROLlary 1.3. With the above notations we have:

$$
\begin{aligned}
& \max \left\{\mu_{1}^{\mathcal{H}}, \mu_{1}^{\mathcal{K}}\right\}=\mu_{1} \\
& \min \left\{\mu_{\ell_{1}}^{\mathcal{H}}, \mu_{\ell_{2}}^{\mathcal{K}}\right\}=\mu_{\ell}
\end{aligned}
$$

Proof. Obvious.

We will use the well-known refined version of Clifford's theorem: 
TheOREM 1.4. Clifford-plus. Let F be a smooth curve of genus $g$. Let $D$ be a divisor of degree $d$ such that the linear system $|D|$ has dimension $r-1$ and let $\phi_{|D|}: F \rightarrow \mathbb{P}^{r-1}$ be the rational map associated to $D$. Then it holds :

(i) if $d \leq 2 g-2$ then $d \geq 2 r-2$;

(ii) if $\operatorname{deg}\left(\phi_{|D|}\right)=m$ then $d \geq m(r-1)$;

(iii) assume that $\operatorname{deg} \phi_{|D|}=1$; then there are two cases: a) if $d \leq g-1$ then $d \geq 3 r-4 ; b)$ if $d \geq g$ then $d \geq \frac{1}{2}(3 r+g-4)$.

Moreover if there exists a divisor $D^{\prime}$ on the smooth curve $C$ and a double cover $\sigma: F \rightarrow C$ such that $|D|=Z+\sigma^{\star}\left|D^{\prime}\right|$ ( $Z$ is the fixed part of $|D|)$ then $d \geq 2(r-1+g(C))$.

\section{Proof. See [4, Lemme 5.1]}

Assume that $\mathcal{O}_{S}(H)$ is an invertible sheaf on $S$ such that $\mathcal{E}=f_{\star} \mathcal{O}_{S}(H)$ is a rank $g$, locally free sheaf on $B$. Let $0=\mathcal{E}_{0} \subset \mathcal{E}_{1} \subset \cdots \subset \mathcal{E}_{l}=\mathcal{E}$ be its Harder-Narasimhan filtration. We consider $\eta \in \operatorname{Pic}(B)$ a sufficiently ample sheaf such that $\mathcal{E}_{i}(\eta)=\mathcal{E}_{i} \otimes \eta$ is globally generated and the rational map $\rho_{i}: S \rightarrow \mathbb{P}\left(\mathcal{E}_{i}\right)$ induced by the natural sheaf homomorphism $f^{*} \mathcal{E}_{i} \rightarrow f^{*} f_{*} \mathcal{O}_{S}(H) \rightarrow \mathcal{O}_{S}(H)$. Let $\sigma: \tilde{S} \rightarrow S$ be the elimination of the indeterminacy of $\rho_{i}$ for every $i$ and let $\mathbb{P}_{i}$ be the sublinear system of $\left|\sigma *\left(H+f^{\star} \eta\right)\right|$ such that $\mathbb{P}_{i}=\mathbb{P}\left(H^{0}\left(B, \mathcal{E}_{i}(\eta)\right)\right)$ for $i=1, \ldots, l$. Xiao defined the following divisors on $\tilde{S}$ : $Z_{i}$ which is the fixed part of $\mathbb{P}_{i}, M_{i}=\sigma * H-Z_{i}$ which is the moving part of $\mathbb{P}_{i}$ and $N_{i}=M_{i}-\mu_{i} F$. By [14] $N_{i}$ is a nef $\mathbb{Q}$ divisor for every $i$. We point out that the restriction $\mathbb{P}_{i_{\mid F}}$ gives a sublinear system of $\left|H_{\mid F}\right|$ of dimension at least $r_{i}=\operatorname{rk}\left(\mathcal{E}_{i}\right)$, with fixed part $Z_{i_{\mid F}}$ and moving part $M_{i_{\mid F}}$ of degree $d_{i}=N_{i} F$. It is easy to see that these definitions do not depend on $\eta$. Thus we can give the following definition:

Definition 1.5. We call $\left\{M_{i \mid F}, r_{i}, d_{i}\right\}$ the Xiao's data associated to the sheaf $\mathcal{E}=f_{\star} \mathcal{O}_{S}(H)$.

Proposition 1.6. Let $f: S \rightarrow B$ be a fibration with general fibre $F$. Let $H$ be a divisor on $S$ and suppose there are a sequence of effective divisors on a suitable blow up $\sigma: \tilde{S} \rightarrow S, Z_{1} \geq Z_{2} \cdots \geq Z_{l} \geq Z_{l+1}=0$ and 
a sequence of rational numbers $\mu_{1}>\mu_{2}>\cdots>\mu_{l} \geq \mu_{l+1}=0$ such that for every $i, N_{i}=\sigma^{*} H-Z_{i}-\mu_{i} F$ is a nef $\mathbb{Q}$-divisor then

$$
H^{2} \geq \sum_{i=1}^{l}\left(d_{i}+d_{i+1}\right)\left(\mu_{i}-\mu_{i+1}\right)
$$

where $d_{i}=N_{i} F$.

Proof. See [17, Lemma 2].

Xiao's method is to combine 1.6 and 1.4 if $H=K_{S / B}$ or if $H$ induces on the general fibre $F$ a sublinear system of $\left|K_{F}\right|$.

\section{$\S 2$. The slope of double covers}

In this section we will be concerned with the following objects:

Definition 2.1. Let $f: S \rightarrow B$ be a relatively minimal fibration. We say that $f$ is a double cover fibration (double cover, for short) if there exists a relatively minimal fibration $\phi: V \rightarrow B$ and a rational map $\pi$ : $S---\rightarrow V$ over $B$ which is a generically two to one map. Otherwise we say that $f$ is a non double cover fibration (non double cover for short).

Roughly speaking, double cover fibrations correspond to the curves with an involution in the theory of curves. Of course, if $f$ is a double cover fibration then $F$ is a double cover. The converse is not true. In [1] Example 1.2 a bielliptic fibration is given for which a non trivial base change is needed in order to be a double cover fibration. In [3] is proved that if the general fibre $F$ is a double cover of a curve of fixed genus $\gamma$ in a unique way, then the corresponding involution glues to a global involution of $S$ and so $f$ itself is a double cover. It is easy to check that if $g \geq 4 \gamma+2$ such condition holds.

Let $f$ be a double cover fibration. We have

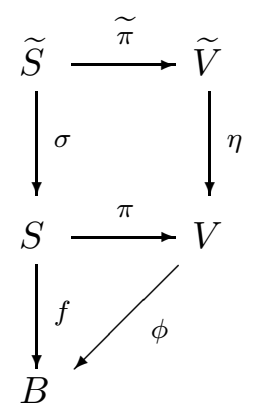


where $\phi$ and $\pi$ exist by definition, $\pi$ is a generically 2-to-1 rational map and $\phi$ is a relatively minimal fibration; $\eta: \widetilde{V} \rightarrow V$ and $\sigma: \widetilde{S} \rightarrow S$ are any birational maps such that the induced rational map $\tilde{\pi}=\eta^{-1} \circ \pi \circ \sigma$ is a morphism. Let $\widetilde{f}=f \circ \sigma$ and $\widetilde{\phi}=\phi \circ \eta$. Note that at general $t \in B$ $f^{-1}(t)=\widetilde{f}^{-1}(t), \phi^{-1}(t)=\widetilde{\phi}^{-1}(t)$. The map $\eta \circ \widetilde{\pi}$ factorizes by Stein Theorem as $\eta \circ \widetilde{\pi}=\pi_{0} \circ u$, where $u: \widetilde{S} \rightarrow S_{0}$ is birational and $\pi_{0}: S_{0} \rightarrow V$. Let $\mathcal{L} \in \operatorname{Pic}(V)$ such that $\mathcal{L}^{\otimes 2}=\mathcal{O}_{V}(R)$ where $R$ is the branching divisor of $\pi_{0}$. By standard theory of cyclic coverings we know that

$$
\begin{aligned}
f_{*} \omega_{S / B}=\widetilde{f}_{*} \omega_{\widetilde{S} / B}=\phi_{*}\left((\eta \circ \widetilde{\pi})_{*} \omega_{\widetilde{S} / B}\right) & =\phi_{*}\left(\omega_{V / B} \oplus\left(\omega_{V / B} \otimes \mathcal{L}\right)\right) \\
& =\phi_{*} \omega_{V / B} \oplus \phi_{*}\left(\omega_{V / B} \otimes \mathcal{L}\right) .
\end{aligned}
$$

We will refer to the following remark:

Remark 2.2. Let $\mathcal{H}=\phi_{*} \omega_{V / B}, \mathcal{K}=\phi_{*}\left(\omega_{V / B} \otimes \mathcal{L}\right)$. Let $q_{f}=q(S)-$ $b, s_{1}=q(V)-b$ and $s_{2}=q_{f}-s_{1}$. According to Fujita's decomposition (see[8]) we have $f_{*} \omega_{S / B}=\mathcal{E} \oplus \mathcal{O}_{B}^{\oplus s}$. A simple computation shows that then we obtain $\mathcal{H}=\mathcal{F} \oplus \mathcal{O}_{B}^{\oplus s_{1}}, \mathcal{K}=\mathcal{G} \oplus \mathcal{O}_{B}^{\oplus s_{2}}$ and $\mathcal{E}=\mathcal{F} \oplus \mathcal{G}$.

In order to estimate a lower bound for the slope in the double cover case the following is the key technical result. The first part is due to Konno (cf. [12]). We reproduce here a proof for lack of a suitable reference.

Proposition 2.3. Let $f: S \rightarrow B$ be a non isotrivial, minimal genus $b$ pencil of curves of genus $g$ which is a double cover of a genus $\gamma \geq 1$ fibration $\phi: V \rightarrow B$. Maintain the notations of 2.2. Let $0 \subset \mathcal{H}_{1} \subset \ldots \subset \mathcal{H}_{\ell_{1}-1} \subset \mathcal{H}_{\ell_{1}}$, $\left\{\left(M_{i \mid E}^{\mathcal{H}}, r_{i}^{\mathcal{H}}, d_{i}^{\mathcal{H}}\right)\right\}_{i=1}^{\ell_{1}}$ and $0 \subset \mathcal{K}_{1} \subset \ldots \subset \mathcal{K}_{l_{2}-1} \subset \mathcal{K}_{l_{2}},\left\{\left(M_{i \mid E}^{\mathcal{K}}, r_{i}^{\mathcal{K}}, d_{i}^{\mathcal{K}}\right)\right\}_{i=1}^{l_{2}}$ be the Harder Narasimhan filtration and the Xiao data of $\mathcal{H}$ and $\mathcal{K}$ respectively. Set $\chi_{f}=\operatorname{deg}\left(f_{*} \omega_{S / B}\right), \chi_{1}=\operatorname{deg} \mathcal{H}$ and $\chi_{2}=\operatorname{deg} \mathcal{K}$. Assume $g \geq 2 \gamma+1$. Then

(i) $K_{S / B}^{2}-4 \chi \geq-4\left(\mu_{1}^{\mathcal{H}}+\mu_{l}^{\mathcal{H}}\right)+2(g-2 \gamma+1) \max \left\{\frac{\mu_{1}^{\mathcal{H}}}{\gamma}, \mu_{l}^{\mathcal{H}}\right\}$. In particular if $g \geq 4 \gamma+1$ then $\lambda(f) \geq 4$.

(ii) $K_{S / B}^{2} \geq \frac{8 g(g-1)}{g^{2}+g-1} \chi_{1}$.

(iii) If $g \geq 2 \gamma+s_{2}$ then

$$
K_{S / B}^{2} \geq 4 \frac{(g-1)\left(g-s_{2}-1\right)}{(g-1)(g-\gamma)-s_{2} g} \chi_{2}
$$




$$
\begin{aligned}
& \text { If } g \leq 2 \gamma+s_{2} \text { then } \\
& \qquad K_{S / B}^{2} \geq 8 \frac{g(g-1)}{\left.g^{2}+g-1\right)} \chi_{2} .
\end{aligned}
$$

Proof. We follow the well-known construction of Horikawa for the canonical resolution of a double cover. We have obtained $\eta \circ \widetilde{\pi}: \widetilde{S} \rightarrow V$ a generically 2-to-1 morphism from a blow-up of $S$ onto a relatively minimal genus $b$ pencil of genus $\gamma$. Now we consider

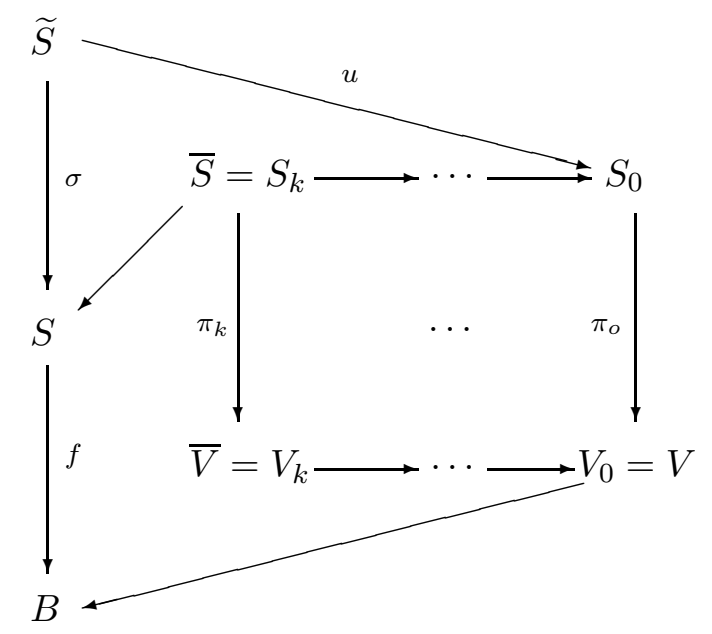

where: $\bullet \eta \circ \tilde{\pi}=\pi_{0} \circ u$ is the Stein factorization of $\eta \circ \tilde{\pi}$, with $u$ birational, $\pi_{0}$ finite (so it is a double cover) and $S_{0}$ normal. $V_{0}$

- $\pi_{k}: S_{k} \rightarrow V_{k}$ is the canonical resolution of singularities of $\pi_{0}: S_{0} \rightarrow$

- $\bar{\sigma}: S_{k} \rightarrow S$ is the birational morphism defined by the relative minimality of $f$. The maps $\pi_{0}: S_{0} \rightarrow V_{0}$ and $\pi_{k}: S_{k} \rightarrow V_{k}$ are determined by divisors $R_{0}$ on $V_{0}, R_{k}$ on $V_{k}$ and line bundles $\mathcal{L}_{0}, \mathcal{L}_{k}$ such that $\mathcal{L}_{0}^{\otimes 2}=\mathcal{O}_{V_{0}}\left(R_{0}\right)$, $\mathcal{L}_{k}^{\otimes 2}=\mathcal{O}_{V_{k}}\left(R_{k}\right)$. First of all we have

$$
\begin{aligned}
K_{S / B}^{2}-4 \chi_{f} & =\left(K_{S}^{2}-4 \chi \mathcal{O}_{S}\right)-4(b-1)(g-1) \\
& \geq\left(K_{\bar{S}}^{2}-4 \chi \mathcal{O}_{\bar{S}}\right)-4(b-1)(g-1) .
\end{aligned}
$$

For smooth double covers $\pi_{k}: \bar{S} \rightarrow \bar{V}$ we have

$$
\begin{aligned}
\chi \mathcal{O}_{\bar{S}} & =2 \chi \mathcal{O}_{\bar{V}}+\frac{1}{2} \mathcal{L}_{k} K_{\bar{V}}+\frac{1}{2} \mathcal{L}_{k} \mathcal{L}_{k} \\
K \frac{2}{S} & =2 K \frac{2}{V}+4 \mathcal{L}_{k} K_{\bar{V}}+2 \mathcal{L}_{k} \mathcal{L}_{k}
\end{aligned}
$$


so we have

$$
K_{\bar{S}}^{2}-4 \chi \mathcal{O}_{\bar{S}}=2\left[K_{V_{k}}^{2}-4 \chi \mathcal{O}_{V_{k}}\right]+2 \mathcal{L}_{k} K_{V_{k}}
$$

By the canonical resolution of singularities of $\pi_{0}: S_{0} \rightarrow V_{0}$ we obtain

$$
2\left[K_{V_{k}}^{2}-4 \chi \mathcal{O}_{V_{k}}\right]+2 \mathcal{L}_{k} K_{V_{k}} \geq 2\left[K_{V_{0}}^{2}-4 \chi \mathcal{O}_{V_{o}}\right]+2 \mathcal{L}_{0} K_{V_{0}}
$$

(i) By (1), (2) and (3) we have that

$$
K_{S / B}^{2}-4 \chi_{f} \geq 2\left(K_{V / B}^{2}-4 \chi_{1}\right)+K_{V / B} R
$$

where $R=R_{0}$ is the branch divisor of $S_{0} \rightarrow V_{0}=V$. We want to estimate the term $K_{V / B} R$.

By $\left[17\right.$, Lemma 3] we have a nef $\mathbb{Q}$-divisor $N_{1}$ and an effective divisor $Z_{1}$ in $V$ such that $K_{V / B} \equiv N_{1}+\mu_{1}^{\mathcal{H}} E+Z_{1}$, where $E$ is the fibre of $\phi: V \rightarrow B$. Let $R=R_{h}+R_{v}$ be the decomposition of $R$ in its horizontal and vertical part respectively. Let $R_{h}=C_{1}+\ldots+C_{m}$ be the decomposition into its irreducible components (note that $R$ is reduced since $S_{0}$ is normal). Set $n_{i}$ the multiplicity of $C_{i}$ in $Z_{1}$. Then

$$
\sum_{i=1}^{m} n_{i} C_{i} E \leq Z_{1} E \leq 2(h-1)
$$

since $E$ is nef and $Z_{1} \leq K_{V / B}$.

Hurwitz formula yields

$$
2(g-2 h+1)=R_{h} E=\sum_{i=1}^{m} C_{i} E
$$

By construction

$$
\left(n_{i}+1\right) K_{V / B}-\mu_{1}^{\mathcal{H}} E \equiv n_{i}\left(K_{V / B}+C_{i}\right)+N_{1}+\left(Z_{1}-n_{i} C_{i}\right)
$$

and it follows

$$
\left(\left(n_{i}+1\right) K_{V / B}-\mu_{1}^{\mathcal{H}} E\right) C_{i} \geq 0
$$

since $\left(K_{V / B}+C_{i}\right) C_{i} \geq 0$ (Hurwitz formula), $N_{1} C_{i} \geq 0$ ( $N_{i}$ is nef) and $\left(Z_{1}-n_{i} C_{i}\right) C_{i} \geq 0\left(C_{i}\right.$ is not a component of $\left.Z_{1}-n_{i} C_{i}\right)$. 
Claim. $K_{V / B} R \geq \frac{2(g-2 \gamma+1)}{h} \mu_{1}^{\mathcal{H}}$.

Proof of the Claim. We can assume $n_{1} \geq n_{2} \geq \ldots \geq n_{m} \geq 0$.

If $h-1 \geq n_{1}\left(\geq n_{i}\right.$ for all $\left.i\right)$ then, by $(6),\left(h K_{V / B}-\mu_{1}^{\mathcal{H}} E\right) C_{i} \geq 0$ since $K_{V / B}$ is nef.

Assume $h \leq n_{1}$. Since $n_{1} C_{1} E \leq 2(h-1)$ then $C_{1} E=1$. Note that (4) gives $n_{i} \leq 2 h-2-n_{1}$ for $i \geq 2$. Hence, using (5) and (6) we have

$$
\begin{aligned}
K_{V / B} R_{h} & \geq \mu_{1}^{\mathcal{H}} \sum_{i=1}^{m} \frac{1}{n_{i}+1} C_{i} E \\
& \geq \mu_{1}^{\mathcal{H}}\left(\frac{C_{1} E}{n_{1}+1}+\frac{\left(R_{h}-C_{1}\right) E}{2 h-1-n_{1}}\right)=\mu_{1} \mathcal{H}\left(\frac{1}{n_{1}+1}+\frac{2 g-4 h+1}{2 h-1-n_{1}}\right) \\
& \geq \mu_{1} \frac{2(g-2 h+1)}{h}
\end{aligned}
$$

since $n_{1} \geq h$. This proves the Claim.

Finally, since $K_{V / B}-\mu_{\ell_{1}}^{\mathcal{H}} E$ is nef we have by (5)

$$
K_{V / B} R \geq 2(g-2 h+1) \mu_{\ell_{1}}^{\mathcal{H}}
$$

In $[17$, p.460] Xiao gives the following bound for any fibration:

$$
K_{V / B}^{2} \geq 4 \chi_{1}-2\left(\mu_{1}^{\mathcal{H}}+\mu_{\ell_{1}}^{\mathcal{H}}\right)
$$

and if we recall that $K_{S / B}^{2}-4 \chi_{f} \geq 2\left(K_{V / B}^{2}-4 \chi_{1}\right)+K_{V / B} R$ we obtain the desired inequality:

$$
K_{V / B}^{2}-4 \chi_{f} \geq-4\left(\mu_{1}^{\mathcal{H}}+\mu_{\ell_{1}}^{\mathcal{H}}\right)+2(g-2 \gamma+1) \max \left\{\frac{\mu_{1}^{\mathcal{H}}}{h}, \mu_{\ell_{1}}^{\mathcal{H}}\right\} .
$$

(ii) We consider the Xiao's data $\left\{\left(M_{i \mid E}^{\mathcal{H}}, r_{i}^{\mathcal{H}}, d_{i}^{\mathcal{H}}\right)\right\}_{i=1}^{\ell_{1}}$. Since $\left|M_{i \mid E}^{\mathcal{H}}\right|$ is a sublinear system of $\left|K_{E}\right|$, by Clifford's lemma $d_{i}^{\mathcal{H}} \geq 2\left(r_{i}^{\mathcal{H}}-1\right)$. By pullback it induces on $F$ a linear system of degree $a_{i} \geq 4\left(r_{i}^{\mathcal{H}}-1\right)$. Hence, for $1 \leq i \leq \ell_{1}-1$ we have $a_{i}+a_{i+1} \geq 8 r_{i}^{\mathcal{H}}-4$, and for $i=\ell_{1}, a_{\ell_{1}}+a_{\ell_{1}+1} \geq$ $4 r_{\ell_{1}}-4+2 g-2 \geq 8 h-4$ since $g \geq 2 h+1$ by hypothesis. By proposition 1.6 we obtain

$$
K_{S / B}^{2} \geq 8 \sum_{i=1}^{\ell_{1}} r_{i}^{\mathcal{H}}\left(\mu_{i}^{\mathcal{H}}-\mu_{i+1}^{\mathcal{H}}\right)-4 \mu_{1}^{\mathcal{H}}=8 \chi_{1}-4 \mu_{1}^{\mathcal{H}} \geq 8 \chi_{1}-4 \mu_{1}^{\mathcal{E}}
$$


since $\mu_{1}^{\mathcal{E}} \geq \mu_{1}^{\mathcal{H}}$. Hence by [10, Lemma 2.7] (see also Theorem 3.1 (ii)) we have: $\left(1+\frac{2 g-1}{g(g-1)}\right) K_{S / B}^{2} \geq 8 \chi_{1}$.

(iii) Now we want to compare $K_{S / B}^{2}$ with $\chi_{2}$. Let $N_{i}^{\mathcal{K}}$ and $Z_{i}^{\mathcal{K}}$ be the divisor on $V$ (on a suitable blow up of $V$ ) associated to the HarderNarasimhan decomposition of $\mathcal{K}$ (We assume $\pi: S \rightarrow V$ for simplicity). We put $N_{i}=\pi^{*}\left(N_{i}^{\mathcal{K}}\right), Z_{i}=\pi^{*}\left(Z_{i}^{\mathcal{K}}\right), H=K_{S / B}, \mu_{i}=\mu_{i}^{\mathcal{K}}$ where $i=1, \ldots, l_{2}$. Since $H_{i}=H-Z_{i}-\mu_{i} F=\pi^{*}\left(K_{V / B}+\mathcal{L}-Z_{i}^{\mathcal{K}}-\mu_{i}^{\mathcal{K}} E\right)$ then $H_{i}$ is nef and by $1.6 H^{2} \geq \sum_{i=1}^{l_{2}}\left(d_{i}+d_{i+1}\right)\left(\mu_{i}-\mu_{i+1}\right)$ where $d_{i}=N_{i} F=2 d_{i}^{\mathcal{K}}, i=1, \cdots, l_{2}$. We consider Xiao's data for $\mathcal{K}:\left\{\left(M_{i \mid E}^{\mathcal{K}}, r_{i}^{\mathcal{K}}, d_{i}^{\mathcal{K}}\right)\right\}_{i=1}^{l_{2}}$. Now the linear systems $\left|M_{i \mid E}^{\mathcal{K}}\right|$ are sub-linear systems of $\left|K_{E}+\mathcal{L}_{\mid E}\right|$, so not always they are special.

We put $r_{i}=r_{i}^{\mathcal{K}}$. We have: $d_{i}^{\mathcal{K}} \geq 2\left(r_{i}^{\mathcal{K}}-1\right)$ if $r_{i}^{\mathcal{K}} \leq \gamma$ and $d_{i}^{\mathcal{K}}=r_{i}^{\mathcal{K}}+\gamma-1$ if $r_{i}^{\mathcal{K}} \geq \gamma-1$. We distinguish two cases: $g \geq 2 \gamma+s_{2}$ or $g \leq 2 \gamma+s_{2}$.

First Case: $g \geq 2 \gamma+s_{2}$. If we consider the degree as a function of the rank we easily see that $d_{i}^{\mathcal{K}} \geq \frac{g-s_{2}-1}{g-s_{2}-\gamma-1}$. Thus

$$
d_{i}^{\mathcal{K}}+d_{i+1}^{\mathcal{K}} \geq 2 \frac{g-s_{2}-1}{g-s_{2}-\gamma-1} r_{i}-\frac{g-s_{2}-1}{g-s_{2}-\gamma-1}
$$

if $i \leq l_{2}-1$ and $d_{l_{2}}^{\mathcal{K}}+d_{l_{2}+1}^{\mathcal{K}} \geq 2 \frac{g-s_{2}-1}{g-s_{2}-\gamma-1}\left(g-s_{2}-\gamma\right)-2 \frac{g-s_{2}-1}{g-s_{2}-\gamma-1}$. We put $A=\frac{g-s_{2}-1}{g-s_{2}-\gamma-1}$ and $B=2 \frac{g-s_{2}-1}{g-s_{2}-\gamma-1}$, then

$$
K_{S / B}^{2} \geq \sum_{i=1}^{l_{2}}\left(d_{i}+d_{i+1}\right)\left(\mu_{i}-\mu_{i+1}\right) \geq \sum_{i=1}^{l_{2}}\left(4 A r_{i}-B\right)\left(\mu_{i}-\mu_{i+1}\right)-B \mu_{l_{2}}
$$

that is: $K_{S / B}^{2} \geq 4 A \chi_{2}-B\left(\mu_{1}+\mu_{l_{2}}\right)$. We recall that $K_{S / B}^{2} \geq d_{l_{2}}\left(\mu_{1}+\mu_{l_{2}}\right)$; then

$$
\left(1+\frac{2 g-2-2 s_{2}}{(2 g-2)\left(g-\gamma-s_{2}-1\right)} K_{S / B}^{2} \geq 4 A \chi_{2}\right.
$$

so: $K_{S / B}^{2} \geq 4 \frac{(g-1)\left(g-s_{2}-1\right)}{(g-1)(g-\gamma)-s_{2} g} \chi_{2}$.

Second Case: $g \leq 2 \gamma+s_{2}$.

If $g \leq 2 \gamma+s_{2}$ then $d_{i} \geq 4\left(r_{i}-1\right)$, so $K_{S / B}^{2} \geq 8 \chi_{2}-4 \mu_{1}$ then by $[10$, Lemma 2.7] (see also 3.1 (ii)) we have

$$
K_{S / B}^{2} \geq 8 \frac{g(g-1)}{g^{2}+g-1} \chi_{2}
$$


In the next two theorems we find lower bounds for $\lambda(f)$ in the case of double covers, considering or not the influence of the relative irregularity of $f$ (see $\S 3$ ). Our bound-functions $l=l\left(g, \gamma, s_{1}, s_{2}\right)$ or $\widetilde{l}=\widetilde{l}(g, \gamma)$ have rather complicate expressions to be able to check their sharpness. Nevertheless we can give examples to check their asymptotic good behaviour.

TheOREM 2.4. Let $f: S \rightarrow B$ be a non isotrivial minimal genus $b$ pencil of curves of genus $g$ which is a double cover of a fibration of genus $\gamma$. We maintain the notations of 2.2 .

(i) If $g \geq 2 \gamma+s_{2}$ and $g>4 \gamma+1$ then

$$
\begin{aligned}
& \lambda(f) \geq \\
& \quad 4+4 \frac{(g-4 \gamma-1)\left[(g-1)(\gamma-1)+s_{2}\right]}{(g-4 \gamma-1)\left[(g-1)(g-\gamma)-g s_{2}\right]+2(g-1)\left(g-s_{2}-1\right)\left(\gamma-s_{1}\right) \gamma} .
\end{aligned}
$$

(ii) If $4 \gamma+1 \leq g \leq 2 \gamma+s_{2}$ then

$$
\lambda(f) \geq 4+8 \frac{2(g-4 \gamma-1)(g-3}{2(g-4 \gamma-1)+8(g-1)\left(\gamma-s_{1}\right) \gamma} .
$$

Proof. Looking independently to the cases $\mu_{\ell_{1}}^{\mathcal{H}} \geq \mu_{1}^{\mathcal{H}} / \gamma$ and $\mu_{\ell_{1}}^{\mathcal{H}} \leq \mu_{1}^{\mathcal{H}} / \gamma$ in 2.3 we always get

$$
K_{S / B}^{2}-4 \chi \geq \frac{2(g-4 \gamma-1)}{\gamma} \mu_{1}^{\mathcal{H}}
$$

Since $\mu_{1}^{\mathcal{H}} \geq \frac{1}{\gamma-s_{1}} \chi_{1}$ and $g \geq 4 \gamma+1$ then

$$
K_{S / B}^{2} \geq y_{1}=4 \chi+\frac{2(g-4 \gamma-1)}{\gamma\left(\gamma-s_{1}\right)} \chi_{1}
$$

By Proposition 2.3 we know that if $g \geq 2 \gamma+s_{2}$ then

$$
K_{S / B}^{2} \geq y_{2}=4 \frac{(g-1)\left(g-s_{2}-1\right)}{(g-1)(g-\gamma)-s_{2} g} \chi-4 \frac{(g-1)\left(g-s_{2}-1\right)}{(g-1)(g-\gamma)-s_{2} g} \chi_{1}
$$


The bounds given above and in Proposition 2.3 can be viewed as linear functions of $\chi_{1}=x$. We consider the region delimited by this two linear inequalities in the plane $(x, y)$. Since $y_{2}\left(x_{0}\right)=y_{1}\left(x_{0}\right)$ implies

$$
x_{0}=\frac{\left[4 g(\gamma-1)+1-\gamma+s_{2}\right]\left(\gamma-s_{1}\right) \gamma}{2\left[(g-1)(g-\gamma)-g s_{2}\right](g-4 \gamma-1)+4(g-1)\left(g-s_{2}-1\right)\left(\gamma-s_{1}\right) \gamma} \chi
$$

then we find

$$
\begin{aligned}
\lambda(f) & \geq 4+ \\
\quad 4 & \frac{(g-4 \gamma-1)\left[g(\gamma-1)+1-\gamma+s_{2}\right]}{(g-4 \gamma-1)\left[(g-1)(g-\gamma)-g s_{2}\right]+2(g-1)\left(g-s_{2}-1\right)\left(\gamma-s_{1}\right) \gamma} .
\end{aligned}
$$

The same proof holds if $g \leq 2 \gamma+s_{2}$.

In an easier way we prove the theorem 0.5 stated in the introduction:

TheOREM 2.5. Let $f: S \rightarrow B$ be a non isotrivial minimal genus $b$ pencil of curves of genus $g$ which is a double cover of a fibration of genus $\gamma$. If $g \geq 4 \gamma+1$ then

$$
\lambda(f) \geq 4+4 \frac{(\gamma-1)(g-4 \gamma-1)}{(g-4 \gamma-1)(g-\gamma)+2(g-1) \gamma^{2}} .
$$

Theorem 2.4 has the following nice corollary:

Corollary 2.6. If $q(S)=q(V)=b+\gamma$ and $g \geq 2 \gamma+1$ then

$$
\lambda(f) \geq 4 \frac{g-1}{g-\gamma} .
$$

Proof. It follows from 2.4 since $s_{2}=0, s_{1}=s=\gamma$.

Remark 2.7. We notice that if $\gamma=1$ we find $\lambda(f) \geq 4$ for bielliptic fibrations of genus $g \geq 5$; see [1].

We give now some examples that show that the bounds given are asymptotically sharp.

ExAMPLE 2.8. Let $A$ be an abelian surface with simple base points linear system $|C|, C^{2}=4$. Then $\mathrm{g}(\mathrm{C})=3$. Take $C_{1}, C_{2}$ two smooth and transversal members, let $\sigma: \widetilde{A} \rightarrow A$ be the blow-up at the 4 base points and let $E$ be the proper transform of $C$; then $E$ is the fibre of a fibration 
$\tau: \widetilde{A} \rightarrow \mathbb{P}^{1}$. Let $Z=Z_{1}+Z_{2}+Z_{3}+Z_{4}$ be the $\sigma$-exceptional reduced divisor. Note that $\tau(Z)=\mathbb{P}^{1}$. Let $n>>m>>0$ and $D=n E+m Z$; then $|2 D|$ has no base point. We can take then a smooth member $R \in|2 D|$ and we consider the associated double cover $\pi: S \rightarrow \widetilde{A}$ which has an induced fibration $f_{n, m}: S \rightarrow \mathbb{P}^{1}$. The general fibre $F$ is a double cover of $E$ and we have (note that $K_{\widetilde{A}}=\sigma^{*} K_{A}+Z=Z$ )

$$
\begin{aligned}
& \gamma=g(E)=3 \\
& g=g(F)=4 m+5 \\
& K_{S}^{2}=2\left(K_{\widetilde{A}}+D\right)^{2}=8(m+1)(2 n-m-1) \\
& \chi \mathcal{O}_{S}=2 \chi \mathcal{O}_{\widetilde{A}}+\frac{1}{2} D K_{\widetilde{A}}+\frac{1}{2} D^{2}=2 m(2 n-m)+2(n-m)
\end{aligned}
$$

By Kawamata-Viehweg vanishing theorem, $h^{1}\left(\widetilde{A}, \mathcal{O}_{\widetilde{A}}(-D)\right)=0$ since $D$ is nef and big; hence

$$
q(S)=q(\widetilde{A})=2
$$

and the slope is:

$$
\lambda\left(f_{n, m}\right)=\frac{8(m+1)(2 n-m-1)+32(m+1)}{2 m(2 n-m)+2(n-m)+8(m+1)} .
$$

For each fixed $m>0$ we have $g=4 m+5, \gamma=3$ and if we allow $n$ to be arbitrarily big $\lambda\left(f_{n, m}\right)$ tends to

$$
\frac{16(m+1)}{4 m+2}=4+\frac{8}{g-3}=\lambda^{\prime} .
$$

Now if in Theorem 2.4 we put the data of $f_{n, m}: s_{2}=0, s_{1}=2, \gamma=3$, $g=4 m+5$ and this time we allow $m$ to increase, then we obtain

$$
\lambda(f) \geq 4+\frac{8}{(g-3)+\frac{6(g-1)}{g-13}} \simeq 4+\frac{8}{g+3} .
$$

Remark 2.9. Let $Y$ be a smooth surface and let $B$ be a smooth curve and denote by $\pi_{Y}: Y \times B \rightarrow Y, \pi_{B}: Y \times B \rightarrow B$ the two natural projections. Consider $C, E \in \operatorname{Div}(Y)$ where $E$ has genus $\gamma$ and let $D, G \in \operatorname{Div}(B)$ such that there exist $W, V$ smooth divisors, $W \in\left|2\left(\pi_{Y}^{*}(C)+\pi_{B}^{*}(D)\right)\right|$ and $V$ $\in\left|\pi_{Y}^{*}(E)+\pi_{B}^{*}(G)\right|$. Let $\tau: Z \rightarrow Y \times B$ be the double cover branched on $W$ and set $S=\tau^{*}(V)$. We put $\pi=\tau_{\mid S}, \phi=\pi_{B \mid V}, f=\phi \circ \pi$ and we 
assume that $W_{\mid V}=\Delta$ is a smooth divisor. Then $S$ is smooth, $f: S \rightarrow B$ is a fibration and if $m=\operatorname{deg}(G), n=\operatorname{deg}(D)$ and $g$ is the genus of the general fibre $F$ of $f$ then

$$
\lambda(f)=6 \frac{4(g-1)(n+m)+2 m\left(K_{Y}+C+E\right)^{2}}{(3 n+6 m)(g-1)+12 m \chi\left(\mathcal{O}_{Y}\right)+6 m(g(C)-1)+3 n E C}
$$

If we consider a $K 3$ surface $Y$ we find that for each smooth curve $B$ and for each $\gamma \geq 2$ there exists a double cover fibration $f$ of genus $g=4 \gamma-3$ such that $\lambda(f)=\frac{(16 n+32 m)(\gamma-1)}{(3 n+5 m)(\gamma-1)+4}$. If $B=\mathbb{P}^{1}$ we can take $n=m=1$ and obtain a genus 5 fibration $f: S \rightarrow \mathbb{P}^{1}$ with $\lambda(f)=4$ which is a double cover of a genus 2 fibration. In particular the slope 4 can be achieved by double cover fibrations with $\gamma>1$.

Remark 2.10. The constant $4 \frac{g-1}{g-\gamma}$ that appears in Corollary 2.6 plays a curious role in the study of double covers: it appears as a limit bound when adding fibres to the ramification locus. Indeed, let $F$ and $E$ be respectively, the general fibre of the fibrations $f: S \rightarrow B, \phi: V \rightarrow B$ where $f$ is assumed to be relatively minimal and non isotrivial. To simplify we assume (although it is not necessary) that $\pi: S \rightarrow V$ is a double covering branched on a smooth divisor $\Delta \in|2 \mathcal{L}|$, where $\mathcal{L} \in \operatorname{Pic}(V)$ such that $f=\pi \circ \phi$. Let $\Delta_{n} \in\left|2\left(\mathcal{L}+\phi^{*} D_{n}\right)\right|$ where $D_{n}$ is a divisor on $B$ of degree $n>0$, let $\pi_{n}: S_{n} \rightarrow V$ be the double covering branched on $\Delta_{n}$ and $f_{n}=\pi_{n} \circ \phi$. Then the sequence of the slopes $\left\{\lambda\left(f_{n}\right)\right\}_{n \geq 0}$ is monotonous and $\lim _{n \rightarrow \infty} \lambda\left(f_{n}\right)=$ $4 \frac{g-1}{g-\gamma}$.

In particular, if $\gamma=0$ or 1 (hyperelliptic or bielliptic fibrations) we obtain $4-\frac{4}{g}$ and 4 respectively, which are the exact lower bounds (cf. [17], [1]). Nevertheless is not true that $\lambda_{\exp }=4 \frac{g-1}{g-\gamma}$ is in general a lower bound for double cover fibrations, as the following example shows.

Example 2.11. We start as in [4, 2.6 Example 3]. Let $Y=A \times H$ where $A$ and $H$ are elliptic curves and $\epsilon$ is a point of order two on $A$. Let $X=Y /\langle\sigma\rangle$ where $\sigma$ is an involution defined on $Y$ by $\sigma(a, h)=(a+\epsilon,-h)$. We denote by $A^{\prime}$ the quotient of $A$ by the group $\{0, \epsilon\}$ and by $h_{1}, \ldots, h_{4}$ the points of order 2 on $H$. Let $p: X \rightarrow A^{\prime}$ and $q: X \rightarrow \mathbb{P}^{1}=B$ the two natural elliptic fibrations on $X$. Clearly $K_{X}=p^{*}(\eta)$ where $\eta$ is the divisor on $\operatorname{Pic}^{0}\left(A^{\prime}\right)$ associated to the étale covering $A \rightarrow A^{\prime}$. Let $A_{i}^{\prime}=q^{-1}\left(h_{i}\right)$ for $i=1,2,3,4$ and $Q \in A^{\prime}$. Since the divisor $\delta=p^{*}(\eta+d Q)+A_{1}^{\prime}$ is 2-divisible on $X$ we consider the double covering $\mu: V \rightarrow X$ associated to $\delta$. We denote 
$p \circ \mu=\phi: V \rightarrow B$ and $q \circ \mu=l: X \rightarrow \mathbb{P}^{1}$. Let $C=\phi^{*}\left(P^{\prime}\right)$ and $E=l^{*}(P)$ where $P^{\prime} \in A^{\prime}$ and $P \in B$. Set $\mathcal{L}=n E+m C$. Let $\pi: S_{d, n, m} \rightarrow V$ be the double covering associated to $\mathcal{L}$ and let $f_{d, n, m}: S_{d, n, m} \rightarrow B$ be the induced fibration on $B$ with fibre $F=\pi^{*}(E)$. By the standard theory of double covering we have :

$$
\lambda\left(f_{d, n, m}\right)=8 \frac{2 n m+2 n d+5 m+5 d}{4 n m+2 n d+5 m+6 d}
$$

and $g=g(F)=4 m+4 d+1, \gamma=g(E)=2 d+1$. In particular $\lambda_{\exp }=$ $4 \frac{g-1}{g-\gamma}=8 \frac{m+d}{2 m+d}=\lim _{n \rightarrow \infty} \lambda\left(f_{d, n, m}\right)$ but $\lim _{d \rightarrow \infty} \lambda_{\exp }=8>8 \frac{2 n+5}{2 n+6}=$ $\lim _{d \rightarrow \infty} \lambda\left(f_{d, n, m}\right)$.

We remark that, in this counterexample, to obtain $\lambda(f)<\lambda_{\exp }$ we have $g \sim 2 \gamma$, and that $F$ is a double cover of a double cover.

Our techniques requires the assumption $g \geq 4 \gamma+1$. On the other hand our next theorem (0.6 in the introduction) shows that for a double cover fibration $\lambda(f) \geq 4$ holds with a few exceptions.

Theorem 2.12. Let $f: S \rightarrow B$ be a non isotrivial minimal genus $b$ pencil of curves $F$ of genus $g$ which is a double cover of a fibration $\sigma: V \rightarrow$ $B$ with fibre $E$ of genus $\gamma$. If $F$ is not hyperelliptic nor tetragonal, $\gamma \geq 1$ and $g \geq 2 \gamma+11$ then $\lambda(f) \geq 4$.

Proof. By [1, Theorem 2.1] we can assume $F$ is not bielliptic since $2+11=13 \geq 6$. We can also assume $F$ is not trigonal otherwise $\lambda(f) \geq$ $\frac{14(g-1)}{3 g+1} \geq 4$ if $g \geq 9$ using [11] (Main theorem).

Consider the Harder Narasimhan filtration of $\mathcal{E}=f_{*} \omega_{S / B}: 0=\mathcal{E}_{0} \subset \mathcal{E}_{1} \subset$ $\ldots \subset \mathcal{E}_{l}=\mathcal{E}$ with slopes $\mu_{1}>\ldots>\mu_{l} \geq 0$ and Xiao's data $\left\{\left(M_{i \mid F}, r_{i}, d_{i}\right)\right\}_{i=1}^{l}$. Note that if $\left|M_{i \mid F}\right|$ induces a map $\phi_{i}$ we have :

If $\operatorname{deg}\left(\phi_{i}\right)=1 d_{i} \geq 3 r_{i}-4$ (if $\left.d_{i} \leq g-1\right), d_{i} \geq \frac{3 r_{i}+g-4}{2}$ (otherwise);

If $\operatorname{deg}\left(\phi_{i}\right)=2 d_{i} \geq 2 r_{i}+2$ (since $F$ is not hyperelliptic nor bielliptic)

If $\operatorname{deg}\left(\phi_{i}\right)=3 d_{i} \geq 3 r_{i}$ (since $F$ is not trigonal)

If $\operatorname{deg}\left(\phi_{i}\right) \geq 4 d_{i} \geq 4\left(r_{i}-1\right)$.

Observe that, since $M_{i} \leq M_{i+1}$, the map $\phi_{i}$ factorizes through $\phi_{i+1}$ and then $d_{i+1} \mid d_{i}$. Note also that $r_{i+1} \geq r_{i}+1$ and $d_{i+1} \geq d_{i}$. Then we can prove $d_{i}+d_{i+1} \geq 4 r_{i}+1$ with a few exceptions. Indeed $\left|M_{i}\right|$ does not define any map only if $\left(r_{1}, d_{1}\right)=(1,0)$. Then $d_{2} \geq 5=4 r_{1}+1$ except if $d_{2}=2,3,4$. All these possibilities imply $r_{2}=2$ according to the previous 
inequalities and hence $F$ would be hyperelliptic, trigonal or tetragonal, all of these being impossible by hypothesis. From now on we assume $r_{i} \geq 2$.

If $\operatorname{deg}\left(\phi_{i}\right) \geq 2$ then $d_{i} \geq 2 r_{i}$ and hence $d_{i}+d_{i+1} \geq 2 d_{i}+1 \geq 4 r_{i}+1$, if $d_{i}<d_{i+1}$; if $d_{i}=d_{i+1}$, then $\phi_{i}=\phi_{i+1}$ and hence $d_{i}+d_{i+1} \geq 4 r_{i}+2$.

If $\operatorname{deg}\left(\phi_{i}\right)=1$ then also $\operatorname{deg}\left(\phi_{i+1}\right)=1$. If $d_{i}, d_{i+1} \leq g-1$ then $d_{i}+d_{i+1} \geq$ $3 r_{i}-4+3 r_{i+1}-4 \geq 6 r_{i}-5 \geq 4 r_{i}+1$ since $r_{i} \geq 3$ ( $\phi_{i}$ is birational).

If $d_{i} \leq g-1, d_{i+1} \geq g$ then $d_{i}+d_{i+1} \geq 2 d_{i}+1 \geq 6 r_{i}-7 \geq 4 r_{i}+1$ except if $r_{i}=3$. But then $d_{i}+d_{i+1} \geq(3.3-4)+g=5+g \geq 13 \geq 4 r_{i}+1$ since $g \geq 11$ by hypothesis.

Finally assume $d_{i}, d_{i+1} \geq g$, being $\phi_{i}$ and $\phi_{i+1}$ birational maps. Then

$$
d_{i}+d_{i+1} \geq \frac{3 r_{i}+g-4}{2}+\frac{3 r_{i+1}+g-4}{2} \geq 3 r_{i}+g-4+\frac{3}{2} \geq 4 r_{i}+1
$$

if $r_{i} \leq g-3$ (the case $r_{i}=g-3$ needs a bit care).

Assume $r_{i}=g-2$. If $r_{i+1}=g$ then $d_{i+1}=2 g-2$ and we are done. If $r_{i+1}=g-1$ then the only case to check is $d_{i}=2 g-5, d_{i+1}=2 g-3$. Note that then $h^{0}\left(F, K_{F}-M_{i_{\mid F}}\right)=h^{0}\left(F, K_{F}-M_{i+1_{\mid F}}\right)=1$ since $F$ is not hyperelliptic. By Riemann-Roch $r_{i}=h^{0}\left(F, M_{i_{\mid F}}\right)=1+d_{i}+1-g=g-3$ which is impossible.

Assume $r_{i}=g-1$. Then $d_{i}=2 g-3,\left(M_{i+1_{\mid} F}, d_{i+1}\right)=\left(r_{l}, d_{l}\right)=$ $(g, 2 g-2)$ and $d_{i}+d_{i+1}=4 g-5=4 r_{i}-1$.

For $r_{i}=g=r_{l}$ we have $d_{l}+d_{l+1}=2 d_{l}=4 g-4=4 r_{l}-4$. By 1.6 we conclude

$$
\begin{aligned}
K_{S / B}^{2} & \geq \sum_{i=1}^{l}\left(d_{i}+d_{i+1}\right)\left(\mu_{i}-\mu_{i+1}\right) \\
& \geq \sum_{i=1}^{l}\left(4 r_{i}+1\right)\left(\mu_{i}-\mu_{i+1}\right)-2\left(\mu_{l-1}-\mu_{l}\right)-5 \mu_{l} \\
& =4 \chi+\mu_{1}-2 \mu_{l-1}-3 \mu_{l}
\end{aligned}
$$

if $r_{l-1}=g-1, d_{l-1}=2 g-3$; otherwise

$$
K_{S / B}^{2} \geq \sum_{i=1} l\left(4 r_{i}+1\right)\left(\mu_{i}-\mu_{i+1}\right)-5 \mu_{l}=4 \chi+\mu_{1}-5 \mu_{l} .
$$

Let us consider first the general case. If $\mu_{1} \geq 5 \mu_{l}$ we are done. Assume $\mu_{1} \leq 5 \mu_{l}$. Let $\mathcal{H}=\phi_{*}\left(\omega_{V \mid B}\right)$ and $\mathcal{K}=\phi_{*}\left(\omega_{V \mid B} \otimes \mathcal{L}\right)$. By $1.2, \mathcal{E}_{i}=\mathcal{H}_{\psi(i)} \oplus$ $\mathcal{K}_{\phi(i)}$ where $\mu_{i}=\mu_{\psi(i)}^{\mathcal{H}}=\mu_{\phi(i)}^{\mathcal{K}}$. By 1.3 we have that $\mu_{l}=\min \left\{\mu_{\ell_{1}}^{\mathcal{H}}, \mu_{l_{2}}^{\mathcal{K}}\right\} \leq$ $\mu_{\ell_{1}}^{\mathcal{H}}, \mu_{1}=\max \left\{\mu_{1}^{\mathcal{H}}, \mu_{1}^{\mathcal{K}}\right\} \geq \mu_{1}^{\mathcal{H}}$. Hence $\mu_{1}^{\mathcal{H}} \leq \mu_{1}<5 \mu_{l} \leq 5 \mu_{\ell_{1}}^{\mathcal{H}}$. By 2.3 we have

$$
K_{S / B}^{2} \geq 4 \chi-24 \mu_{\ell_{1}}^{\mathcal{H}}+2(g-2 \gamma+1) \max \left\{\frac{\mu_{1}^{\mathcal{H}}}{\gamma}, \mu_{\ell_{1}}^{\mathcal{H}}\right\}
$$


If $\gamma \geq 5$ or $\frac{\mu_{1}^{\mathcal{H}}}{\gamma} \leq \mu_{\ell_{1}}^{\mathcal{H}}$ we have $\max \left\{\frac{\mu_{1}^{\mathcal{H}}}{\gamma}, \mu_{\ell_{1}}^{\mathcal{H}}\right\}=\mu_{\ell_{1}}^{\mathcal{H}}$ and hence

$$
K_{S / B}^{2} \geq 4 \chi+2(g-2 \gamma-11) \mu_{\ell_{1}}^{\mathcal{H}} \geq 4 \chi
$$

when $g \geq 2 \gamma+11$.

If $\gamma=2,3,4$ and $\frac{\mu_{1}^{\mathcal{H}}}{\gamma} \geq \mu_{\ell_{1}}^{\mathcal{H}}$ then

$$
K_{S / B}^{2} \geq 4 \chi-24 \mu_{\ell_{1}}^{\mathcal{H}}+2(g-2 \gamma+1) \frac{\mu_{1}^{\mathcal{H}}}{\gamma} \geq 4 \chi+2(g-2 \gamma-11) \frac{\mu_{1}^{\mathcal{H}}}{\gamma} \geq 4 \chi
$$

when $g \geq 2 \gamma+11$.

Consider finally the special case $r_{l-1}=g-1, d_{l-2}=2 g-3$. By 1.6, with the notations of 1.6 where $H=\omega_{S / B}$ it follows that $Z_{l-1}$ is a section of $f$ such that $Z_{l-1_{\mid F}} \equiv K_{F}-M_{l-1_{\mid F}}$. We recall that $M_{l-1_{\mid F}}$ is the base point free linear system induced on the general fibre by the piece $\mathcal{E}_{l-1}$. By $1.2 \mathcal{E}_{l-1}=\mathcal{H}_{\psi(l-1)} \oplus \mathcal{K}_{\phi(l-1)}$. Since $r_{l-1}=r_{l}-1$ we only have two possibilities: either $\mathcal{H}_{\psi(l-1)}=\mathcal{H}_{\ell_{1}}, \mathcal{K}_{\phi(l-1)}=\mathcal{K}_{l_{2}-1}$ and $r_{l_{2}-1}^{\mathcal{K}}=g-\gamma-1$ or $\mathcal{H}_{\psi(l-1)}=\mathcal{H}_{\ell_{1}-1}, \mathcal{K}_{\phi(l-1)}=\mathcal{K}_{l_{2}}$ and $r_{\ell_{1}-1}^{\mathcal{K}}=\gamma-1$. We claim the second possibility can not occur. Indeed consider the double cover $\pi_{\mid F}: F \rightarrow E$. We have that

$$
H^{0}\left(F, \omega_{F}\right) \simeq H^{0}\left(E, \omega_{E}\right) \oplus H^{0}\left(E, \omega_{E} \otimes \mathcal{L}_{\mid E}\right)
$$

This decomposition means that if $D$ is the ramification divisor on $F$ and $t \in H^{0}\left(F, \mathcal{O}_{F}(D)\right)$ then for every $\omega \in H^{0}\left(F, \omega_{F}\right), \omega=t \pi_{\mid F}^{*}\left(\omega_{1}\right)+\pi_{\mid F}^{*}\left(\omega_{2}\right)$ where $\omega_{1} \in H^{0}\left(E, \omega_{E}\right)$ and $\omega_{2} \in H^{0}\left(E, \omega_{E} \otimes \mathcal{L}_{\mid E}\right)$.

We have $V \in H^{0}\left(F, \omega_{F}\right)$ a codimension one subspace which produces, after taking out the base point, the linear series $\left|M_{l-1_{\mid F}}\right|$. The second possibility asserts that $V=\pi_{\mid F}^{*} V_{1} \oplus \pi_{\mid F}^{*} V_{2}$ where $V_{2}=H^{0}\left(E, \omega_{E} \otimes \mathcal{L}_{\mid E}\right)$ and $V_{1}$ is a codimension one subspace of $H^{0}\left(E, \omega_{E}\right)$. Since $\operatorname{deg}\left(\omega_{E} \otimes \mathcal{L}_{\mid E}\right) \geq$ $2 \gamma+10, V_{2}$ is base point free. Hence $\pi_{\mid F}^{*} V_{2}$ is base point free: a contradiction since $V$ has a base point.

So we have the following decompositions

$$
\mathcal{E}_{l}=\mathcal{H}_{\ell_{1}} \oplus \mathcal{K}_{l_{2}}, \mathcal{E}_{l-1}=\mathcal{H}_{\ell_{1}} \oplus \mathcal{K}_{l_{2}-1}
$$

where $r_{l_{2}-1}^{\mathcal{K}}=g-\gamma-1$. If $\mathcal{E}_{l-2}=\mathcal{H}_{j} \oplus \mathcal{K}_{k}$ we have several possibilities according to 1.2 .

If $j=\ell_{1}, k=l_{2}-2$ then $\mu_{l-1}=\mu\left(\mathcal{E}_{l-1} / \mathcal{E}_{l-2}\right)=\mu\left(\mathcal{K}_{l_{2}-1} / \mathcal{K}_{l_{2}-2}\right)=\mu_{l_{2}-1}^{\mathcal{K}}$ and $\mu_{\ell_{1}}^{\mathcal{H}}>\mu_{l-1}$. 
If $j=\ell_{1}-1, k=l_{2}-1$ then $\mu_{l-1}=\mu\left(\mathcal{H}_{\ell_{1}} / \mathcal{H}_{\ell_{1}-1}\right)=\mu_{\ell_{1}}^{\mathcal{H}}$.

If $j=\ell_{1}-1, k=l_{2}-2$ then $\mu_{l-1}=\mu_{l_{2}}^{\mathcal{H}}=\mu_{l_{2}-1}^{\mathcal{K}}$.

In any case we get $\mu_{l-1}=\mu_{\ell_{1}}^{\mathcal{H}}$. Since always happens that $\mu_{1} \geq \mu_{1}^{\mathcal{H}}$ and $\mu_{l} \leq \mu_{\ell_{1}}^{\mathcal{H}},(7)$ reads:

$$
K_{S / B}^{2} \geq 4 \chi+\mu_{1}-2 \mu_{l-1}-3 \mu_{l} \geq 4 \chi+\mu_{1}^{\mathcal{H}}-5 \mu_{\ell_{1}}^{\mathcal{H}}
$$

If $\mu_{1}^{\mathcal{H}} \geq 5 \mu_{\ell_{1}}^{\mathcal{H}}$ we are done. If $\mu_{1}^{\mathcal{H}}<5 \mu_{\ell_{1}}^{\mathcal{H}}$ then we can repeat the argument of the general case.

\section{$\S 3$. The slope of non-Albanese fibrations}

In this section we consider the problem of the influence of the relative irregularity $q_{f}=q(S)-b$ on the lower bound of the slope. In case $f$ is a double cover fibration this problem has been solved in the previous section so we will deal only with non double cover fibrations. The nice fact is that we find a lower bound which is an increasing function of the genus $g$ and of $q_{f}$.

If $q_{f}=0$ then the general bound $\lambda(f) \geq 4-\frac{4}{g}$ holds and it is sharp. So we will consider fibrations with $q_{f}=q(S)-b>0$. Those are precisely the fibrations for which the Albanese map of $S$ does not factorize through $f$ (i.e., $b=0$ and $q>0$ or $S$ is of Albanese general type). We call such fibrations non-Albanese fibrations.

The two basic known results in this area are:

TheOREM 3.1. Let $f: S \rightarrow B$ be a non locally trivial minimal genus $b$ pencil of curves of genus $g$.

(i) If $q_{f}=q(S)-b>0$, then $\lambda \geq 4$

(ii) Let $\mu_{1}=\operatorname{deg} \mathcal{E}_{1} / \operatorname{rank}\left(\mathcal{E}_{1}\right)$ where $\mathcal{E}_{1}$ is the maximal semistable subbundle of $f_{*} \omega_{S / B}$. If $g \geq 2$ and $g>q_{f}$ then $K_{S / B}^{2} \geq \frac{4 g(g-1)}{(2 g-1)} \mu_{1}$. In particular $\lambda(f) \geq \frac{4 g(g-1)}{(2 g-1)\left(g-q_{f}\right)}$.

Proof. (i) is [17, Theorem 2.4]. (ii) is [10, Lemma 2.7].

We motivated our Theorem 0.7 in the introduction: here we will give its proof.

THEOREM 3.2. Let $f: S \rightarrow B$ be a minimal genus $b$ pencil of curves of genus $g$ which is not locally trivial and not a double cover fibration. Assume that $g=g(F) \geq 5$ and $q_{f}=q(S)-b \geq 1$ then the following bounds hold: 
(i) If $q_{f} \geq 2$ and $g \geq \frac{3}{2} q_{f}+2$ then

$$
\begin{aligned}
& \lambda(f) \geq \frac{8 g(g-1)\left(4 g-3 q_{f}-10\right)}{8 g(g-1)\left(g-q_{f}-2\right)+3\left(q_{f}-2\right)(2 g-1)} \quad \text { if } F \text { is not trigonal } \\
& \lambda(f) \geq \frac{4 g(g-1)\left(4 g-3 q_{f}-10\right)}{4 g(g-1)\left(g-q_{f}-2\right)+(g-4)(2 g-1)} \quad \text { if } F \quad \text { is trigonal }
\end{aligned}
$$

(ii) If $g<\frac{3}{2} q_{f}+2$ then

$$
\lambda(f) \geq \frac{4 g(g-1)(2 g-7)}{\frac{4}{3} g(g-1)(g-3)+(g-4)(2 g-1)}
$$

Proof. (i), (ii) Consider Fujita's decomposition $f_{*} \omega_{S / B}=\mathcal{A} \oplus \mathcal{Z}$ with $\mathcal{Z}=\mathcal{O}_{B}^{\oplus q_{f}}$ and the Harder-Narasimhan filtration of $\mathcal{A}$ :

$$
0=\mathcal{A}_{0} \subseteq \mathcal{A}_{1} \subseteq \ldots \subseteq \mathcal{A}_{\ell}=\mathcal{A}
$$

As in $\S 1$ we produce nef $\mathbb{Q}$-divisors $N_{i}$, and effective divisors $Z_{i}$ in a suitable blow-up of $S \quad \sigma: \widetilde{S} \rightarrow S$ such that

$$
N_{i}+\mu_{i} F+Z_{i} \equiv N_{j}+\mu_{j} F+Z_{j} \equiv \sigma^{*} K_{S / B}
$$

where $\left\{\mu_{i}\right\}$ are the Harder-Narasimhan slopes of $\mathcal{A}$. Note that we can define $N_{\ell+1}=\sigma^{*} K_{S / B}, Z_{\ell+1}=0, \mu_{\ell+1}=0$. Observe also that, if $r_{i}=\mathrm{rk} \mathcal{A}_{i}$, $\sum_{i=1}^{\ell} r_{i}\left(\mu_{i}-\mu_{i+1}\right)=\operatorname{deg} \mathcal{A}=\operatorname{def} \chi_{f}$.

Each $N_{i}$ induces on $F$ a base point free linear system of degree $d_{i}$ with (projective) dimension greater or equal than $r_{i}-1$. Note that $N_{i}+\mu_{i} F=H_{i}$ is induced by a map $\varphi_{i}: S \rightarrow \mathbb{P}_{B}\left(\mathcal{A}_{i}\right)$ and restricted to fibres, it induces the above linear system. By hypothesis $\varphi_{i}$ is never a double cover onto the image and so the induced map $\psi_{i}$ on the general $F$ is not a double cover. Hence we have

$$
\begin{array}{ll}
d_{i} \geq 3\left(r_{i}-1\right) & \text { if } \operatorname{deg} \psi_{i} \geq 3 \\
d_{i} \geq 3 r_{i}-4 & \text { if } \operatorname{deg} \psi_{i}=1 \text { and } d_{i} \leq g-1 \\
d_{i} \geq \frac{3 r_{i}+g-4}{2} & \text { if } \operatorname{deg} \psi_{i}=1 \text { and } d_{i} \geq g
\end{array}
$$


the latest two inequalities being "Clifford plus" Lemma. Considering the above inequalities in the $(r, d)$-plane, we have the following two possibilities (note that the lines $d=3 r-4$ and $d=\frac{3 r+g-4}{2}$ meet exactly at the point $\left.\left(r=\frac{1}{3}(g+4), d=g\right)\right)$ depending on $\operatorname{rank} \mathcal{A}=g-q_{f}$.

Case 1. $g-q_{f} \geq \frac{1}{3}(g+4)$

In the $(r, d)$-plane $d=\frac{2 g-\frac{3}{2} q_{f}-5}{g-q_{f}-2} r-\frac{g-4}{g-q_{f}-2}$, is the line joining the point $(2,3)$ and the point $\left(g-q_{f}, 2 g-\frac{3}{2} q_{f}-2\right)$. So in this case for every $1 \leq i \leq \ell$, $d_{i} \geq \frac{2 g-\frac{3}{2} q_{f}-5}{g-q_{f}-2} r-\frac{g-4}{g-q_{f}-2}$ except if $\left(r_{1}, d_{1}\right)=(1,0)$. Note that $g-q_{f}-2>0$ since $g \geq \frac{3}{2} q_{f}+2$.

By definition we have $d_{\ell+1}=2 g-2$. So for $1 \leq i \leq \ell$ we get (since $\left.r_{i+1} \geq r_{i}+1\right)$

$$
d_{i}+d_{i+1} \geq \frac{4 g-3 q_{f}-10}{g-q_{f}-2} r_{i}-\frac{3\left(q_{f}-2\right)}{2\left(g-q_{f}-2\right)}=: A r_{i}+B
$$

except if $\left(r_{1}, d_{1}\right)=(1,0)$ and $\left(r_{2}, d_{2}\right)=(2,3)$. In this exceptional case we get

$$
d_{1}+d_{2}-A r_{1}-B=3-A-B=-\frac{g-\frac{3}{2} q_{f}-1}{g-q_{f}-2}
$$

If this happens $F$ is trigonal since it has a linear system of degree 3 and dimension 1.

Applying Xiao's formula we get, in the general case,

$$
\begin{aligned}
K_{S / B}^{2} & \geq \sum_{i=1}^{\ell}\left(d_{i}+d_{i+1}\right)\left(\mu_{i}-\mu_{i+1}\right) \geq \sum_{i=1}^{\ell} A r_{i}\left(\mu_{i}-\mu_{i+1}\right)+\sum_{i=1}^{\ell} B\left(\mu_{i}-\mu_{i+1}\right)= \\
& =A \chi_{f}+B \mu_{1}=\frac{4 g-3 q_{f}-10}{g-q_{f}-2} \chi_{f}-\frac{3\left(q_{f}-2\right)}{2\left(g-q_{f}-2\right)} \mu_{1}
\end{aligned}
$$

Applying again Konno's bound [10]:

$$
K_{S / B}^{2} \geq \frac{4 g(g-1)}{2 g-1} \mu_{1}
$$

we can eliminate $\mu_{1}$ and we get

$$
K_{S / B}^{2} \geq \frac{8 g(g-1)\left(4 g-3 q_{f}-10\right)}{8 g(g-1)\left(g-q_{f}-2\right)+3\left(q_{f}-2\right)(2 g-1)} \chi_{f}
$$


Note that this bound is a strictly increasing function of $q_{f}$ and that $K_{S / B}^{2} \geq 4 \chi_{f}$ if $q_{f} \geq 2$.

In the exceptional case (when $F$ is trigonal) we get $K_{S / B}^{2} \geq A \chi_{f}+B \mu_{1}-\frac{g-\frac{3}{2} q_{f}-1}{g-q_{f}-2}\left(\mu_{1}-\mu_{2}\right) \geq A \chi_{f}+\left(B-\frac{g-\frac{3}{2} q_{f}-1}{g-q_{f}-2}\right) \mu_{1}$.

The same argument using $K_{S / B}^{2} \geq \frac{4 g(g-1)}{2 g-1} \mu_{1}$ yields

$$
K_{S / B}^{2} \geq \frac{4 g(g-1)\left(4 g-3 q_{f}-10\right)}{4 g(g-1)\left(g-q_{f}-2\right)+(g-4)(2 g-1)} \chi_{f}
$$

which is also a strictly increasing function of $h$. In this case we need $q_{f} \geq 4$ to get $K_{S / B}^{2} \geq 4 \chi_{f}$.

Case 2. $g-q_{f} \leq \frac{1}{3}(g+4)$

Let $\overline{q_{f}}=\left[\frac{2}{3} g-\frac{4}{3}\right]$. Under our hypotheses $q_{f} \geq \overline{q_{f}}$, so we can take $\overline{\mathcal{A}}=\mathcal{A} \oplus \mathcal{O}_{B}^{\oplus\left(q_{f}-\overline{q_{f}}\right)}$ instead of $\mathcal{A}$. Hence, according to whether we are in the general or in the special case, we get

$$
\begin{aligned}
K_{S / B}^{2} & \geq \frac{8 g(g-1)\left(4 g-3 \overline{q_{f}}-10\right)}{8 g(g-1)\left(g-\overline{q_{f}}-2\right)+3\left(\overline{q_{f}}-2\right)(2 g-1)} \chi_{f} \\
& \geq \frac{8 g(g-1)(2 g-7)}{\frac{8}{3} g(g-1)(g-3)+(2 g-9)(2 g-1)} \chi_{f} \\
K_{S / B}^{2} & \geq \frac{4 g(g-1)\left(4 g-3 \overline{q_{f}}-10\right)}{4 g(g-1)\left(g-\overline{q_{f}}-2\right)+(g-4)(2 g-1)} \chi_{f} \\
& \geq \frac{4 g(g-1)(2 g-7)}{\frac{4}{3} g(g-1)(g-3)+(g-4)(2 g-1)} \chi_{f}
\end{aligned}
$$

since both expressions are increasing functions of $q_{f}$ and $\overline{q_{f}} \geq \frac{2}{3} g-1$. Note that the second bound is slightly smaller than the first one.

Remark 3.3. In the case (ii) of the theorem 0.7 we could consider that for $1 \leq i \leq \ell, d_{i} \geq 3 r_{i}-4$ and hence $d_{i}+d_{i+1} \geq 6 r_{i}-5$ for $1 \leq i \leq \ell-1$. But for $i=\ell$ we would have $d_{\ell}+d_{\ell+1} \geq 2 d_{\ell}+1 \geq 6 r_{\ell}-7$ which produces

$$
K_{S / B}^{2} \geq 6 \chi_{f}-\left(5 \mu_{1}+2 \mu_{\ell}\right)
$$


Hence using Xiao's inequality with indexes $\{1, \ell\}$

$K_{S / B}^{2} \geq\left(d_{1}+d_{\ell}\right)\left(\mu_{1}-\mu_{\ell}\right)+\left(d_{\ell}+d_{\ell+1}\right) \mu_{\ell} \geq d_{\ell}\left(\mu_{1}+\mu_{\ell}\right) \geq\left(3 g-3 q_{f}-4\right)\left(\mu_{1}+\mu_{\ell}\right)$

we get

$$
K_{S / B}^{2} \geq 6 \frac{3 g-3 q_{f}-4}{3 g-3 q_{f}+1} \chi_{f}
$$

which depends on $q_{f}$ and it is better than (ii) for some special values of $\left(g, q_{f}\right)$ but it is a decreasing function of $q_{f}$.

Nevertheless we point out that case (ii) of the theorem 0.7 is doubtful to happen. Indeed, by a conjecture of Xiao (cf. [18]) the following inequality should hold: $q_{f} \leq \frac{1}{2}(g+1)$. This inequality is true when $b=0$ but is known to be false in general (cf. [15]) although it seems that only the constant term should be modified.

Remark 3.4. In Theorem 3.2 we worked with $\mathcal{Z}=\mathcal{O}_{B}^{\oplus(q(S)-b)}$; but in most parts of the proof we only use that $\operatorname{deg} \mathcal{Z}=0$. Hence, we get the same bounds in (ii) if we define $h$ to be the rank of the degree zero part in Fujita's decomposition of $\mathcal{E}=f_{*} \omega_{S / B}\left(h \geq q(S)-b=q_{f}\right)$. Note that then the argument of Theorem 3.2 (ii) does not work since we do not know whether $\mathcal{Z}$ can be cut in pieces of the length we need. In any case the bound of the previous remark holds for this definition of $h$ (just putting $h$ instead of $\left.q_{f}\right)$.

Remark 3.5. Remember that if $F$ is trigonal we have (cf. [11] and $[16])$ :

$$
\lambda(f) \geq \frac{14(g-1)}{3 g+1}
$$

which is better than Theorem 3.2 for $g \gg q-b$ and that gives $\lambda(f) \geq 4$ if $g \geq 9$.

On the other hand we have:

Remark 3.6. As a function on $g$ (fixing $q_{f}$ ) the bounds contained in 0.7 tend to be 4 when $g$ grows (compare Theorem 3.1 (ii) where this limit is 2$)$.

ExAMPLE 3.7. Let $Y$ be a smooth surface, let $B$ be a smooth curve of genus $b, Z=Y \times B$ and let $\pi_{Y}: Z \rightarrow Y, \pi_{B}: Z \rightarrow B$ be the natural 
projections. If $F \in \operatorname{Div}(Y)$ is smooth of genus $g, D \in \operatorname{Div}(B)$ and there exists an ample and smooth divisor $S \in\left|\pi_{Y}^{*}(F)+\pi_{B}^{*}(D)\right|$ then the slope of the fibration $f: S \rightarrow B$ induced on $S$ by $\pi_{B}$ is

$$
\lambda(f)=\frac{6 g-6+K_{Y}^{2}+K_{Y} F}{\chi\left(\mathcal{O}_{Y}\right)+g-1} .
$$

Now if $\rho: Y=\mathbb{P}(\mathcal{E}) \rightarrow C$ is a ruled surface, $H$ is a section such that $H^{2}=\operatorname{deg}(\mathcal{E})$ and $F \equiv 3 H$ the fibration $f_{m}: S_{m} \rightarrow B(m=\operatorname{deg} D)$ has slope:

$$
\lambda\left(f_{m}\right)=\frac{15 m+16(g(C)-1))}{3 m+2(g(C)-1))}
$$

and verifies that $q_{f}=q\left(S_{m}\right)-b=g(C)$. In particular $\lambda\left(f_{m}\right) \geq 5$ and $\lim _{m \rightarrow \infty} \lambda\left(f_{m}\right)=5$. This result shows the non obvious result that for any $q_{f}$, a general lower bound of $\lambda(f)$ is below 5 .

From Theorem 3.2 we deduce Theorem 0.8 in the introduction which shows that $\lambda(f)$ controls the existence of other fibrations on $S$ :

Theorem 3.8. Let $f: S \rightarrow B$ be a minimal genus b pencil of curves of genus $g$ which is not locally trivial and not a double cover fibration. Let $\mathcal{C}=\left\{\pi_{i}: S \rightarrow C_{i} \text { fibrations, } c_{i}=g\left(C_{i}\right) \geq 2, \pi_{i} \neq f\right\}_{i \in I}$. Assume $\mathcal{C} \neq \emptyset$ and let $c=\max \left\{c_{i} \mid i \in I\right\}$. If $q_{f}=q-b \geq 1$ (i.e., $f$ is not an Albanese fibration) then

$$
\lambda(f) \geq 4+\frac{c-1}{g-c} .
$$

Moreover if $\operatorname{dim} \operatorname{alb}(S)=1$ (then necessarily $b=0$ ) we have

$$
\lambda(f) \geq 4+\frac{q-1}{g-q} .
$$

Proof. We recall that if $f$ is not an Albanese fibration then either $\operatorname{dim} \operatorname{alb}(S)=2$ or $b=0$ (provided $q(S) \neq 0$ ).

Let $\pi: S \rightarrow C$ be the fibration with maximal base genus $c \geq 2$ (if $\operatorname{dim} \operatorname{alb}(S)=1$, then $c=q$ and $\pi=$ alb).

Since both the fibre of $f$ and $\pi$ are connected and $g(C) \geq 2$ then $f^{*} \operatorname{Pic}^{0}(B)$ does not include $\pi^{*} \operatorname{Pic}^{0}(C)$. In particular we can choose for $n \gg 0$, a $n$-torsion element $\mathcal{L} \in \operatorname{Pic}^{0}(C)$ such that $\pi^{*} \mathcal{L}^{\otimes i} \notin f^{*} \operatorname{Pic}^{0}(B)$ for 
$1 \leq i \leq n-1$. From the base change $\alpha: \widetilde{C} \rightarrow C$ we can construct the following diagram:

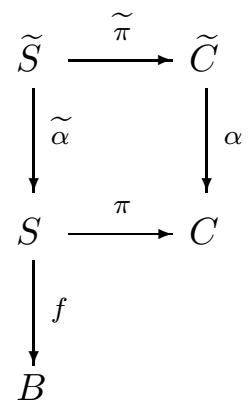

We set $\tilde{f}=f \circ \widetilde{\alpha}$. Since $\mathcal{L}_{\mid F}^{\otimes i} \neq \mathcal{O}_{F}$ for $1 \leq i \leq n-1, \tilde{f}$ has connected fibres and so $\widetilde{f}$ is again a fibration over $B$. Let $\widetilde{F}$ be the fibre of $\widetilde{f}$. Then if $\widetilde{g}=g(\widetilde{F})$,

$$
\widetilde{g}-1=n(g-1)
$$

Moreover we have

$$
q(\widetilde{S})=h^{1}\left(\widetilde{S}, \mathcal{O}_{\widetilde{S}}\right)=h^{1}\left(S, \mathcal{O}_{S}\right)+\sum_{i=1}^{n-1} h^{1}\left(S,\left(\pi^{*} \mathcal{L}^{-i}\right)\right)
$$

From the exact sequence

$$
0 \longrightarrow H^{1}\left(B, \mathcal{L}^{-i}\right) \longrightarrow H^{1}\left(S, \pi^{*} \mathcal{L}^{-i}\right) \longrightarrow H^{0}\left(B,\left(R^{1} \pi_{*} \mathcal{O}_{S}\right) \otimes \mathcal{L}^{-i}\right) \longrightarrow 0
$$

and using that $h^{0}\left(B,\left(R^{1} \pi_{*} \mathcal{O}_{S}\right) \otimes \mathcal{L}^{-i}\right)=0$ except for a finite number of sheaves $\mathcal{L}^{-i} \in \operatorname{Pic}^{0}(C)$ (which can be avoided with the election of $\mathcal{L}$ (see $[5$, Lemma 3.1] and $[2, \S 3])$ we get

$$
\widetilde{q}_{f}=q(\widetilde{S})-b=q(S)-b+(n-1)(c-1)=q_{f}+(n-1)(c-1)
$$

since $h^{1}\left(B, \mathcal{L}^{-i}\right)=c-1$ by Riemann-Roch. In particular, $\widetilde{q_{f}} \geq 2$ if $n \geq 2$.

It is easy to check that if $F$ is trigonal then $\widetilde{F}$ is not trigonal if $n \gg 0$ (see for example [2, Lemma 5.12] ). On the other hand

$$
\lim _{n \rightarrow \infty} \frac{\widetilde{g}}{\widetilde{q_{f}}}=\frac{g-1}{c-1} \geq 2
$$

since the map $\pi_{\mid F}: F \rightarrow C$ is at least of degree two (if it were of degree 1 clearly $F \cong C$ and $S=B \times C$ ). Hence if $n \gg 0$ the case $\widetilde{g}<\frac{3}{2} \widetilde{q_{f}}+2$ can not occur. 
So if $n \gg 0$ we are under the hypotheses of Theorem 3.2 (i) (non trigonal case). On the other hand the slope is invariant under étale base changes (cf. [17]), then we get

$$
\lambda(f)=\lambda(\widetilde{f}) \geq \frac{8 \widetilde{g}(\widetilde{g}-1)\left(4 \widetilde{g}-\widetilde{q_{f}}-14\right)}{8 \widetilde{g}(\widetilde{g}-1)\left(\widetilde{g}-\widetilde{q_{f}}-3\right)+5\left(\widetilde{q_{f}}-2\right)(2 \widetilde{g}-1)}
$$

where $\widetilde{g}=n(g-1)+1, \widetilde{q_{f}}=q_{f}+(n-1)(c-1)$ and $n \in \mathbb{N}$ is arbitrarily large. So we can take limit as $n$ grows and we get

$$
\lambda(f) \geq 4+\frac{c-1}{g-c}
$$

In case $\operatorname{dim} \operatorname{alb}(S)=1$ then clearly $c=q$. Note that if this happens and $b \geq 1$, then $\operatorname{alb}(S)=B$ by the universal property of Albanese variety.

Corollary 3.9. Let $f: S \rightarrow B$ be as in Theorem 3.8. Assume $\lambda(f)<$ $4+\frac{1}{g-2}$. Then $S$ has no other fibration onto a curve $C$ with $g(C)=c \geq 2$.

Corollary 3.10. Let $S$ be a minimal surface with $q(S) \geq 2$ and let $F \subseteq S$ be an irreducible curve of geometric genus $g$ such that $h^{0}\left(S, \mathcal{O}_{S}(F)\right) \geq$ 2. Let $f: \widetilde{S} \rightarrow \mathbb{P}^{1}$ be a relatively minimal fibration with fibre $F$. If $F$ is not a double cover and $\lambda(f)<4+\frac{q-1}{g-q}$ then $S$ is of Albanese general type.

Acknowledgements. We want to thank Professor Kazuhiro Konno who kindly communicated to us the result in Proposition 2.3 (i). The second author would like to thank also all the staff of the Departament de Matemàtica Aplicada I, Universitat Politècnica de Catalunya for the extremely warm hospitality received during his visit in February 1998.

\section{REFERENCES}

[1] M. A. Barja, On the slope of bielliptic fibrations, to appear in the Proc. of the A.M.S.

[2] - On the slope and geography of fibred surfaces and threefolds, Ph. D. Thesis. University of Barcelona, 1998.

[3] M.A. Barja and J.C. Naranjo, Extension of maps defined on many fibres, Collect Math., 49, 2-3 (1998), 227-238.

[4] A. Beauville, L'application canonique pour les surfaces pour les surfaces de type général, Invent. Math., 55 (1979), 121-140.

[5] _ Annullation du $H^{1}$ et systèmes paracanoniques sur les surfaces, J. Reine Math., 388 (1988), 149-157. 
[6] F. Catanese, Fibred surfaces, varieties isogenous to a product and related moduli spaces, to appear American Journal Math, 1-42.

[7] Z. Chen, On the bound of the slope of a non-hyperelliptic fibration of genus 4, Intern. J. Math.,, 4, No.3 (1993), 367-378.

[8] T. Fujita, On Kaehler fibre spaces over curves, J. Math. Soc. Japan, 30 (1978), 779-794.

[9] G. Harder and M. S. Narasimhan, On the cohomology groups of moduli spaces of vector bundles on curves, Math. Ann., 212 (1974), 215-248.

[10] K. Konno, On the irregularity of special non-canonical surfaces, Publ. Res. Inst. Math. Sci., 30 (1994), 671-688.

[11] K. Konno, A lower bound of the slope of trigonal fibrations, Internat. J. Math., 7 no. 1 (1996), 19-27.

[12] K. Konno, A note on a question by M.A. Barja, personal communication (1997).

[13] K. Konno, Clifford index and the slope of fibred surfaces, J. Algebraic Geom., 8 no.2 (1999), 207-220.

[14] N. Nakayama, Zariski decomposition problem for pseudo effective divisors, Proceedings of the meeting and the workshop "Algebraic Geometry and Hodge Theory", vol I, Hokkaido University Math. preprint series.

[15] G. Pirola, On a conjecture of Xiao, J. Reine angew. Math., 431 (1992), 75-89.

[16] Z.E. Stankova-Frenkel, Moduli of trigonal curves, Preprint (1997).

[17] G. Xiao, fibred algebraic surfaces with low slope, Math. Ann., 276 (1987), 449-466.

[18] G. Xiao, Irregularity of surfaces with a linear pencil, Duke Math. J., 55, no. 3 (1987), 597-602.

Miguel Ángel Barja

Departament de Matmática Aplicada I

Universitat Politécnica de Catalunya

Diagonal 647

08028 Barcelona

Spain

barja@ma1.upc.es

Francesco Zucconi

Dipartimento di Matematica e Informatica

Universitá degli studi di Udine

Via delle Scienze, 206

33100 Udine

Italy

zucconi@dimi.uniud.it 